\title{
EFEITOS DA INSTABILIDADE DA TAXA DE CÂMBIO NO COMÉRCIO SETORIAL ENTRE BRASIL E SEUS PRINCIPAIS PARCEIROS COMERCIAIS
}

\author{
Geraldo Moreira Bittencourt * \\ Antônio Carvalho Campos ${ }^{\dagger}$
}

\begin{abstract}
Resumo
Este trabalho teve por objetivo avaliar como a instabilidade cambial tem afetado os fluxos de exportações e importações setoriais do Brasil com seus principais parceiros (China, EUA, Argentina, Holanda, Japão, Alemanha e Chile), no período de 1989 a 2011. Foram realizadas estimações de uma equação gravitacional para as exportações e importações setoriais entre o Brasil e os parceiros considerados. Os resultados para os fluxos de importações e exportações revelaram que a instabilidade cambial e o efeito third country (instabilidade cambial de um terceiro país) são prejudiciais para todos os setores, evidenciando que os dois fluxos de comércio setorial entre o Brasil e seus principais parceiros comerciais são negativamente afetados não só pela própria incerteza cambial, mas também pela instabilidade da taxa de câmbio dos parceiros.
\end{abstract}

Palavras-chave: Instabilidade cambial; Comércio internacional; Modelo gravitacional.

\begin{abstract}
This study aimed to evaluate how the exchange rate instability has affected the flow of sectoral exports and imports of Brazil with its main partners (China, USA, Argentina, Netherlands, Japan, Germany and Chile), in the period 1989-2011. Estimations were carried by a gravity equation for exports and imports, per sectors, between Brazil and its trading partners considered. The results for the flow of imports and exports showed that the effect of exchange rate instability and the third country effect (exchange rate instability of a third country) are detrimental to all sectors, indicating that the two sectoral trade flows between Brazil and its main partners are negatively affected not only by their own exchange rate uncertainty, but also by the instability of the exchange rate of the partners.
\end{abstract}

Keywords: Exchange rate instability; International trade; Gravity model. JEL classification: F13, F19, E39

DOI: http://dx.doi.org/10.1590/1413-8050/ea503

\footnotetext{
* Professor do Departamento de Economia da Universidade Federal de Juiz de Fora/ Campus Governador Valadares (UFJF/GV). Doutorando em Economia Aplicada pela Universidade Federal de Viçosa (UFV). E-mail: geraldo.bittencourt@ufjf.edu.br

† Professor Titular do Departamento de Economia Rural da UFV. E-mail: accampos@ufv.br
} 


\section{Introdução}

A taxa de câmbio é considerada uma das variáveis mais relevantes de uma economia aberta em seu relacionamento com o exterior. Desde o colapso do Sistema Bretton Woods, ${ }^{1}$ em 1971, grande parte dos países desenvolvidos e industrializados passou a adotar o regime de taxas de câmbio flutuante, que ganhou impulso com a abertura e a integração financeira dos mercados. Já na década de 1990, esse processo se acelerou ainda mais, alcançando os países emergentes.

No entanto, esse aumento da integração no mercado financeiro, a difusão do sistema de câmbio flutuante e a onda de liberalização comercial da década de 1980 e início de 1990 expuseram países desenvolvidos e em desenvolvimento a grandes oscilações (instabilidades) nas taxas de câmbio e consequentes incertezas cambiais. Como resultado, os efeitos dessa instabilidade cambial $^{2}$ sobre o investimento e comércio vêm, cada vez mais, tornando-se interesse particular para muitos pesquisadores e formuladores de políticas.

De acordo com Côté (1994), a instabilidade cambial pode ter um efeito negativo direto sobre o comércio internacional, por meio da incerteza e dos custos de ajustamento e, indireto, por meio de seu efeito sobre a alocação de recursos e políticas governamentais. Desse modo, se os movimentos da taxa de câmbio não são totalmente esperados, um aumento na variabilidade cambial pode levar agentes econômicos avessos ao risco a reduzirem suas atividades no comércio mundial.

Por outro lado, a evidência empírica em apoio à hipótese de uma relação negativa entre instabilidade cambial e comércio é mista. De acordo com o estudo de Dellas \& B. (1993), as instabilidades cambiais resultariam em grande risco que, ao invés de inibir os agentes econômicos indiferentes ao risco a comercializarem, acabariam gerando oportunidades de diversificar seu portfólio de risco e aumentarem a expectativa por maiores lucros. Segundo os autores, isso ocorreria, principalmente, em países desenvolvidos dotados de um mercado financeiro altamente eficiente.

Adicionalmente, ao estudar os efeitos da incerteza cambial sobre o comércio, é importante fazer a distinção entre mudanças de curto, médio e longo prazos nas taxas de câmbio. Segundo Peree \& Steinherr (1989), embora a curto prazo o risco da taxa de câmbio possa ser coberto com sucesso nos mercados financeiros, a incerteza além de um horizonte temporal de um ano não pode ser coberta com baixo custo, evidenciando, dessa forma, que a instabilidade cambial de longo prazo constitua um provável problema para os fluxos comerciais internacionais.

No Brasil, o caráter interdependente das políticas monetária, fiscal e cambial, em junção com a instabilidade macroeconômica vivida nos anos de 1980 e início dos anos de 1990, provocou, por várias vezes, modificações na política cambial. Com a implementação do Plano Real e a estabilização dos preços, a partir de 1994, foi introduzido o regime de bandas cambiais, objetivando in-

\footnotetext{
${ }^{1} \mathrm{O}$ sistema consagrado em Bretton Woods estabeleceu o dólar como moeda internacional e esta era a única moeda que manteria sua conversibilidade em relação ao ouro. As outras moedas nacionais eram livremente conversíveis em dólar a uma taxa de câmbio fixa (Vasconcellos et al. 1996).

${ }^{2}$ Pelo fato de as medidas de instabilidade da taxa de câmbio real serem usadas como proxies da incerteza cambial, as expressões instabilidade, volatilidade e incerteza são usadas para descrever o mesmo fenômeno neste estudo.
} 
crementar a credibilidade da política cambial por meio da adoção de medidas que implicavam maior estabilidade da taxa de câmbio real e o padrão de variação da taxa nominal de câmbio. Entretanto, em janeiro de 1999, a manutenção desse regime tornou-se insustentável, levando o governo a adotar um regime de taxas de câmbio flexíveis, gerando uma sensível desvalorização das taxas de câmbio nominal e real. Desde então, sucederam-se fases de estabilidade cambial e de grande variabilidade (incerteza) e desalinhamento da taxa de câmbio (Souza \& Hoff 2003).

Com isso, pode-se dizer que a instabilidade macroeconômica mundial e os diversos planos e políticas econômicas de estabilização adotados ao longo do tempo e implementados pelo Brasil foram responsáveis por grande parte da variabilidade da taxa de câmbio real de médio e longo prazo existente na economia brasileira. Esse raciocínio está associado ao fato de que grandes alterações na taxa de câmbio podem aumentar o grau de incerteza entre agentes econômicos domésticos e seus parceiros comerciais.

Desse modo, de acordo com Kafle (2011), a natureza incerta da taxa de câmbio tornou-se um problema relevante no âmbito da estimativa dos comportamentos comerciais e dos volumes de comércio existentes entre países exportadores e importadores, mostrando que a questão da dimensão do efeito da instabilidade cambial sobre o comércio é um tema que demanda uma importante investigação empírica.

No caso do Brasil, o comércio exterior tem alcançado números recordes de exportação e importação, sendo a China o maior importador de produtos nacionais, seguida pelos Estados Unidos da América (EUA), Argentina, Holanda, Japão, Alemanha e Chile. Já em relação às importações domésticas, os EUA, China, Argentina e Alemanha destacaram-se como os principais países de origem das importações brasileiras (Associação de Comércio Exterior do Brasil 2012).

De acordo com dados estatísticos da Uncomtrade (2012) e conforme a Classificação Uniforme para o Comércio Internacional (CUCI) deve-se destacar que esses sete países (Alemanha, Argentina, Chile, China, Japão, Holanda e EUA) enquadram-se no grupo dos principais parceiros comerciais do Brasil no agregado e por setor, ressaltando que, nos últimos 20 anos, eles representaram expressivas participações no fluxo comercial (exportações e importações) setorial com o Brasil, e essas participações foram: mais de $60 \%$ do fluxo comercial no setor de Máquinas e Equipamentos de Transporte; $50 \%$ no setor de Manufaturados; $60 \%$ dos fluxos no setor de Metais e Minerais; $45 \%$ no setor Agropecuário e mais de $50 \%$ dos fluxos comerciais no setor Químico. ${ }^{3}$

Por outro lado, deve-se destacar que esses parceiros comerciais também passaram por momentos de instabilidade econômica e consequentes flutuações de preços e taxa de câmbio, o que afeta o comércio e a alocação de investimentos. Nesse sentido, pode-se citar o caso da crise cambial do Sistema Monetário Europeu em 1992-1993 e a recente crise da dívida pública na zona do EURO, a crise cambial da Argentina em 2001, ano em que o governo argentino resolveu por fim à paridade do peso/dólar americano, a política de desvalorização da moeda chinesa, que tem gerado controvérsias entre os países, a crise financeira global de 2008, entre outros casos que acabam evidenci-

\footnotetext{
${ }^{3} \mathrm{~A}$ especificação dos setores analisados neste estudo encontra-se no tópico 4.3 , referente à fonte de dados.
} 
ando a necessidade de uma análise do impacto da instabilidade cambial sobre o comércio entre o Brasil e seus principais parceiros.

Diante do exposto, considerando um cenário de incertezas cambiais e de evolução da participação brasileira no comércio internacional, o presente estudo consiste em responder a seguinte questão: a instabilidade da taxa de câmbio de médio e longo prazo, no período de 1989 a 2011,4 afetou o nível das importações e exportações brasileiras com seus principais parceiros comerciais, por setor, uma vez corrigidas pelas variáveis gravitacionais?

A hipótese que permeia este trabalho é que a variabilidade da taxa de câmbio de médio e longo prazo tem influenciado negativamente os fluxos comerciais setoriais do Brasil com seus principais parceiros. Pois, conforme De Grauwe \& De Bellefroid (1987), no longo prazo, o mercado de câmbio passa por um "desalinhamento sustentado" (ou seja, incerteza gerando incerteza na memória dos agentes), que não pode ser facilmente protegido e é muito caro para ser coberto. Dessa forma, se os movimentos da taxa de câmbio não são totalmente esperados, um aumento na incerteza cambial de longo prazo pode levar agentes econômicos avessos ao risco a reduzirem suas atividades no comércio mundial.

Estudar os impactos da instabilidade cambial e de outros determinantes do comércio, tais como tarifas, distância entre países, PIB e da incerteza da taxa de câmbio de um terceiro país (efeito third country) $)^{5}$ sobre o comércio internacional do Brasil, mostra-se de extrema relevância para o fornecimento de informações mais precisas aos agentes econômicos envolvidos. Além disso, pode auxiliar na formação de políticas econômicas equilibradas, buscando uma maior coordenação macroeconômica entre o Brasil e seus principais parceiros.

A contribuição deste estudo diferencia-se dos demais, na literatura precedente, pois realiza a análise desagregada da influência da instabilidade cambial sobre o comércio (exportações e importações) setorial de um país emergente, visto que a maioria dos trabalhos na literatura concentra-se na determinação dos efeitos da incerteza cambial sobre os fluxos comerciais agregados (totais: importações + exportações) para países desenvolvidos.

O artigo está estruturado em outras 5 seções, além desta introdução. Nas duas próximas seções, apresentam-se uma discussão teórica que fundamenta a pesquisa. Na quarta, descreve-se a metodologia e os dados utilizados. Na quinta parte, expõe-se os resultados e, por fim, a sexta e última seção contém a conclusão do artigo.

\section{Teoria do comércio internacional e a incerteza cambial}

Há uma vasta literatura que aborda como a incerteza cambial afeta o comércio internacional. $\mathrm{O}$ argumento geral é que uma maior variabilidade das taxas de câmbio (real e nominal) é prejudicial ao comércio entre países. Além disso,

\footnotetext{
${ }^{4}$ Este intervalo de análise abrange o período em que houve o início de uma maior abertura da economia brasileira ao comercio internacional, mudanças nas políticas cambiais e monetárias do Brasil e de seus parceiros, momentos de crescimento e recessão da economia mundial, entre outros acontecimentos que acabaram provocando uma maior instabilidade da taxa de câmbio dos países considerados.

${ }^{5} \mathrm{O}$ efeito third country é uma medida que leva em conta a incerteza da taxa de câmbio real para todos os outros parceiros comerciais, exceto os dois países envolvidos no comércio em análise.
} 
essa literatura informa que o comércio será tão mais prejudicado quanto mais avessos ao risco forem os agentes, isto é, quanto menores forem as oportunidades de hedging contra o risco de câmbio e quanto maior for a parcela das receitas e despesas em moeda externa (Cho et al. 2002).

De acordo com Hooper \& Kohlhagen (1978), no caso das exportações, a instabilidade da taxa de câmbio leva a uma contração na quantidade ofertada, por afetar a proporção do lucro que não é segurada (hedged) pela firma. Em relação às importações, estas são tratadas como fatores usados na produção de bens vendidos no mercado doméstico. Assim, uma elevação na incerteza cambial aumenta a variância do lucro e muda a curva de demanda da firma para esquerda, levando a um declínio nos preços e na quantidade. A dimensão da resposta aumenta com a magnitude da elasticidade-preço da curva de demanda e com o grau de aversão e exposição ao risco.

Muitos estudos empíricos têm tentado estabelecer uma posição sobre essa questão da incerteza cambial e os fluxos comerciais. Mais recentemente, esses estudos estão sendo realizados com base no modelo gravitacional de comércio e fazendo uso de dados em painel. Contudo, a evidência econométrica tem se mostrado ambígua. Por exemplo, De Grauwe \& Skudelny (2000) utilizaram um modelo gravitacional para estudar o impacto da instabilidade da taxa de câmbio sobre o comércio entre os países da União Europeia e os resultados encontrados evidenciaram a existência de uma relação negativa entre a incerteza cambial e o comércio. Por outro lado, ao analisar o comércio agrícola entre a Hungria e os seus parceiros comerciais, Jozsef (2011) identifica um efeito positivo significativo da instabilidade cambial sobre os fluxos comerciais desse setor. Já Rose (2000),avaliou o comércio bilateral para um painel de 186 países, durante o período 1970-1990, e encontrou um pequeno, mas estatisticamente significativo, efeito negativo da volatilidade cambial sobre o comércio.

Os estudos anteriormente citados encontraram evidências da existência de uma relação mista e significativa entre a instabilidade cambial e o fluxo comercial existente entre os países. No entanto, os aperfeiçoamentos feitos por Anderson \& Van Wincoop (2003) mostram que as relações comerciais de dois países não são afetadas apenas pelas variáveis dos países com os quais comercializam, mas também pelas variáveis dos seus parceiros comerciais. Assim, não é apenas a incerteza cambial que afeta o comércio entre o país i e o país j, mas também a instabilidade da taxa de câmbio dos países com os quais o país i e/ou o país j comercializam. Esse efeito da incerteza cambial dos parceiros sobre o comércio é denominado efeito third country (efeito terceiro país). De acordo com Bittencourt (2004), o efeito terceiro país é o impacto da instabilidade da taxa real de câmbio de um terceiro país no comércio bilateral em análise.

De acordo com os resultados de estudos que utilizam o modelo gravitacional, variáveis como as tarifas e o efeito third country também têm gerado importantes contribuições para a redução do comércio entre os países. Baier \& Bergstrand (2001), utilizando um modelo derivado por Krugman, estudaram oimpacto da redução das tarifas sobre o crescimento do comércio para 16 países da OCDE. De acordo com esse trabalho, os autores encontraram que $26 \%$ do crescimento do comércio são explicados pela redução das tarifas. Bittencourt (2004) estimou o impacto do efeito third country sobre o comércio entre os países do MERCOSUL e os coeficientes estimados para essa variávelapresentaram sinal negativo esperado, dado que o aumento na incerteza cambial em um terceiro país afeta negativamente o comércio bilateral. 


\section{O modelo gravitacional}

O embasamento teórico deste estudo encontra-se, também, na teoria que fundamenta o modelo gravitacional. De acordo com essa metodologia, o volume de comércio entre dois parceiros comerciais é uma função crescente de suas rendas, utilizadas como proxies para o tamanho do mercado em cada país e medidas pelo PIB, e decrescente em relação à distância entre eles, geralmente interpretada como proxy dos custos de transporte entre países (Africano \& Magalhães 2005).

Nesse sentido, de acordo com Kume \& Piani (2000), a justificativa teórica mais difundida para a utilização dessa modelagem remonta ao modelo de comércio desenvolvido por Krugman (1980), que se refere à existência de rendimentos crescentes e custos de transporte, que atuam como um incentivo para a concentração da produção próxima a um grande mercado. Como resultado dessa concentração tem-se economias de escala e minimização dos custos de transporte.

Uma extensão proposta por Anderson \& Van Wincoop (2003) e Anderson \& Van Wincoop (2004) tornou-se a abordagem teórica que mais tem sido empregada para dar suporte ao modelo gravitacional. Esse modelo proposto por Anderson e Van Wincoop pode ser considerado como padrão. Além disso, por ser um modelo de análiseex-post, ele tem sido utilizado para verificar a magnitude e os efeitos de diversas variáveis sobre os fluxos comerciais, tais como a incerteza (volatilidade) cambial, o impacto da aplicação de tarifas, diversos custos de transporte, entre outras.

Segundo Mendonça (2011), a inclusão dos índices de resistência multilateral foi a principal contribuição do trabalho de Anderson \& Van Wincoop (2003) ao modelo gravitacional. Conforme os autores, o comércio entre dois países é impactado, entre outras variáveis, pelo comércio entre todos os países com os quais cada país comercializa, por isso, existe a necessidade de controlar o impacto dos índices de resistência multilateral para que os resultados encontrados não sejam viesados.

Os índices de resistência multilateral sintetizam a resistência média ao comércio entre um país e seus parceiros comerciais. Nesse caso, para dada barreira bilateral entre dois países, $i$ e $j$, se as barreiras foram mais altas entre $j$ e seus outros parceiros comerciais, os preços relativos dos bens no país $i$ serão reduzidos, aumentando as importações provenientes de $i$, visto que o país $i$ possui resistência bilateral menor se comparado aos demais parceiros comerciais de $j$. Assim, barreiras mais elevadas enfrentadas por um exportador reduzem a demanda por seus produtos e, assim, o seu preço de oferta (Anderson \& Van Wincoop 2003).

\section{Metodologia e base de dados}

\subsection{Especificações das variáveis e o modelo empírico}

Alguns estudos procuram adicionar outros elementos estruturais no modelo gravitacional para melhor refletir as condições da realidade. No presente trabalho, diante dos objetivos do estudo e da fundamentação teórica do modelo gravitacional, exposta na seção anterior, optou-se por estimar o modelo básico (composto pelas variáveis: produto dos PIB's e distância geográfica entre paí- 
ses) com a inclusão das tarifas, da incerteza (instabilidade) cambial e do efeito third country, cujas metodologias são apresentadas nesta seção.

A instabilidade da taxa de câmbio é decorrente da incerteza, para os agentes econômicos, relacionada às variações na mesma. Para o cálculo dessa variabilidade, diversas medidas de instabilidade ${ }^{6}$ ou incerteza sobre o comportamento da taxa de câmbio real podem ser utilizadas. Desse modo, seguindo indicações dos estudos de Cho et al. (2002), Bittencourt (2004) e Chit et al. (2010), este trabalho utilizará o desvio padrão móvel (MSD) como medida da instabilidade cambial. ${ }^{7}$

Esse método para mensurar a instabilidade da taxa de câmbio é o mais indicado pela literatura anterior. Por exemplo, Dell'ariccia (1999), Rose (2000) e Jozsef (2011) também utilizaram o desvio padrão móvel (ou MSD - Moving Standard Deviation) das diferenças do logaritmo natural da taxa de câmbio bilateral real entre os países i e $\mathrm{j}$ como proxy para a incerteza cambial. O diferencial desse método é a propriedade que ele tem de ser zero se a taxa de câmbio segue uma tendência constante durante o período de tempo especificado. Isto significa que, se a taxa de câmbio segue uma tendência constante, não haverá instabilidade e a taxa de câmbio para um período de tempo futuro torna-se perfeitamente previsível (Kafle 2011). Além disso, essa medida da instabilidade cambial atribui peso maior às observações extremas. Desse modo, quanto mais observações extremas, mais adequadamente representa-se o comportamento dos comerciantes avessos ao risco (Dell'ariccia 1999).

Adicionalmente, deve-se destacar que a taxa de câmbio utilizada neste estudo será a taxa de câmbio real. De acordo com Farrell et al. (1983), o uso da taxa de câmbio real é o mais indicado, pois sérias consequências podem ser verificadas quando a taxa de câmbio nominal é utilizada, isso porque variações nas taxas nominais podem ser superadas por variações nos níveis de preços em nível nacional. ${ }^{8}$ Desse modo, a taxa de câmbio bilateral real para cada fluxo pode ser obtida pela razão entre a taxa de câmbio real de cada parceiro e a taxa de câmbio real brasileira.

De posse dessa taxa de câmbio bilateral real, prossegue-se ao cálculo do desvio padrão móvel (MSD) das diferenças do logaritmo natural da taxa de câmbio bilateral real entre os países $i$ e $j$ no ano $t\left(U_{i j, t}\right)$, que pode ser escrito como:

$$
U_{i j, t}=\sqrt{\frac{\sum_{n=1}^{k}\left(x_{i j, t-n}-\bar{x}_{i j, t}\right)^{2}}{k-1}}
$$

onde $X_{i j, t}$ é a taxa de câmbio bilateral real entre os países $i$ e $j ; x_{i j, t}=\ln \left(X_{i j, t}\right)$ $\ln \left(X_{i j, t-1}\right)$, sendo utilizadas $k$ defasagens em $\operatorname{anos}^{9}\left(k=2,4,6,8\right.$ ou 10). Já $\overline{x_{i j, t}}$ é a média dos valores passados (ou seja, para os últimos $k$ anos) da variável $x_{i j, t}$.

\footnotetext{
${ }^{6}$ Pelo fato das medidas de instabilidade (volatilidade) da taxa de câmbio real serem usadas como proxies da incerteza cambial, as expressões instabilidade, volatilidade e incerteza são usadas para descrever o mesmo fenômeno neste estudo.

${ }^{7}$ Para mais detalhes sobre as medidas de incerteza da taxa de câmbio, ver Kenen \& Rodrik (1986).

${ }^{8}$ Côté (1994) também defende o uso de taxas reais alegando que se o nível de preços falha em se mover em conjunto com a taxa de câmbio, então o risco dos agentes pode aumentar a medida que a volatilidade da taxa de câmbio nominal cai.

${ }^{9}$ O período de tempo é arbitrariamente escolhido para investigar a robustez dos resultados. Entende-se essas $k$ defasagens como uma medida da "memória dos agentes".
} 
Como visto anteriormente, a incerteza cambial que afeta o fluxo de comércio entre dois países não é resultante apenas da volatilidade da taxa de câmbio desses países, sendo que, as variações das taxas de câmbio dos demais parceiros comerciais também afetam tal fluxo de comércio bilateral. Logo, o efeito third country, que é a medida que leva em conta a volatilidade entre essas taxas de câmbio para todos os outros países de determinado conjunto de dados, exceto aquelas dos países i e j envolvidos no fluxo de comércio bilateral em análise, também deve ser incluído no modelo.

O efeito third country, como é chamado na literatura internacional, foi investigado por Dell'ariccia (1999), e Cho et al. (2002), usando uma medida que leva em consideração a instabilidade da taxa de câmbio real para todos os outros países, exceto os dois países envolvidos no comércio em análise. Os trabalhos desses autores utilizaram as participações dos países no comércio total como ponderações para obter a medida do efeito third country. No entanto, o presente estudo seguirá o exemplo de Bittencourt (2004), que propõe uma diferenciação nas ponderações por setores da economia, ou seja, leva em conta as participações no comércio específicas a cada setor como ponderações para todas as combinações de comércio bilateral entre os 8 países considerados, incluindo Brasil, para cada ano da amostra.

Desse modo, segundo Bittencourt (2004), o cálculo da volatilidade da taxa de câmbio real de um terceiro país (efeito third country) é dado por:

$$
U 3_{i j, t}^{g}=\sum_{i \neq j} u_{i j, t} w_{i j, t}^{g}+\sum_{j \neq i} u_{j i, t} w_{j i, t}^{g}
$$

onde $U 3_{i j, t}^{g}$ é a medida, para o setor $g,{ }^{10}$ da instabilidade da taxa de câmbio real de um terceiro país (ou efeito third country), considerando todos os países de uma amostra, exceto os países $i$ e $j$ envolvidos no fluxo comercial em análise; $u_{i j, t}$ e $u_{j t, t}$ são as medidas de instabilidade da taxa de câmbio bilateral real entre os demais países (exceto os países do fluxo em análise), calculada pelo método do desvio padrão móvel (MSD), descrito anteriormente; e $w_{i j, t}^{g} \mathrm{e}$ $w_{j i, t}^{g}$ são as ponderações, as participações no comércio específico de cada setor $(g)$ dos outros parceiros comerciais, ou seja, representa a participação das exportações do país $i$ no total das importações do país $j$ provenientes de todos os países da amostra de dados, no ano $t$ e setor $g$; ou a participação das exportações do país $j$ no total das importações do país $i$ provenientes de todos os países da amostra de dados, no ano $t$ e setor $g$.

Após essa definição das medidas representativas da incerteza da taxa de câmbio real, resta especificar a mensuração das tarifas. A tarifa é uma variável de custo de fácil mensuração, cujos dados são obtidos com maior facilidade para todos os países. Desse modo, o cálculo da variável tarifas $\left(T_{i j, t}^{g}\right)$ é feito por uma média simples das tarifas efetivamente aplicadas entre os países $i$ e $j$, no período $t$, dentro de cada setor, como demonstrado na formula a seguir:

$$
T_{i j, t}^{g}=\frac{\bar{T}_{i j}}{100}+1
$$

\footnotetext{
${ }^{10} g=1, \ldots, 5$, onde 1 é para o setor agropecuário; 2 para o setor químico;3 para o setor de metais e minerais; 4 para o setor de manufaturados e 5 para o setor de máquinas e equipamentos de transportes.
} 
Por fim, a instabilidade da taxa de câmbio bilateral real, o efeito third country e o equivalente tarifário são incluídos como variáveis explicativas na equação gravitacional básica. Assim sendo, a equação gravitacional empírica a ser estimada, separadamente, para os fluxos de exportação e importação de cada setor $(g)$, apresenta a seguinte forma:

$$
\begin{aligned}
\ln \left(X_{i j, t}\right)= & \alpha+\delta_{j}+\gamma_{t}+\beta_{1} \ln \left(y_{j t} y_{i t}\right)+\beta_{2} \ln \left(d_{i j}\right)+ \\
& \beta_{3} \ln \left(T_{i j, t}^{g}\right)+\beta_{4}\left(U_{i j, t}\right)+\beta_{5}\left(U 3_{i j, t}^{g}\right)+\varepsilon_{i j, t}
\end{aligned}
$$

onde $X_{i j, t}$ ) = é o fluxo de comércio (exportação ou importação) setorial entre os países $i$ e $j$ no período $t$; $\alpha=$ é a constante; $\delta_{j}=$ representam os efeitos fixos para os países; $\gamma_{t}=$ representa os efeitos fixos para os anos da amostra; $y_{j t} y_{i t}=$ é o produto das rendas (PIB's) dos países $i$ e $j$ no período $t$ (participação da renda dos países $i$ e $j$ na renda mundial); $d_{i j}=$ é a distância entre os países $i$ e $j ; T_{i j, t}^{g}=$ é a média simples das tarifas incidentes no comércio setorial entre o país $i$ e o parceiro $j$, no ano $t$, mensurada por meio da equação (3); $U_{i j, t}=$ é a medida da instabilidade (volatilidade) da taxa de câmbio bilateral real entre os países $i$ e $j$ no ano $t$, mensurada por meio da equação (1); $U 3_{i j, t}^{g}=$ é a medida do efeito third country, para o setor $g$, no ano $t$, calculado pela equação 2; e $\varepsilon_{i j, t}=$ corresponde ao termo de erro aleatório.

Como na equação gravitacional teórica, espera-se que a variável utilizada para o tamanho do mercado dos países (produto das rendas) possua sinal positivo, pois quanto maior for o mercado (nível de renda) de cada país, maior será o seu poder de atração. O coeficiente da variável distância, por sua vez, deve apresentar sinal negativo, pois se espera que países que possuam maior distância entre si, comercializem cada vez menos, porque o aumento da distância aumenta os custos de transporte e transação, reduzindo o comércio entre os países. Quanto às variáveis representativas da incerteza cambial, espera-se que a instabilidade cambial, seja do país em estudo, seja dos demais parceiros (efeito third country), pelo exposto nas seções anteriores, apresente sinal negativo ou positivo, dependendo do setor e fluxo de comércio em análise. Já as tarifas, como elevam os custos do comércio, assim como a distância, impactarão negativamente no volume comercializado entre os países.

\subsection{Métodos de estimação}

A escolha do método de estimação é de grande relevância para a mensuração dos efeitos das variáveis, em estudo, sobre os fluxos de comércio. Nesse sentido, a forma como as variáveis são adicionadas ao modelo depende do efeito a ser estudado. Conforme Anderson \& Van Wincoop (2004), na estimação de uma equação gravitacional é importante que os termos de resistência multilateral sejam levados em consideração, dessa forma, os autores sugerem que a estimação da equação empírica seja realizada por meio de um modelo de efeitos fixos ${ }^{11}$ por Mínimos Quadrados Ordinários (MQO) utilizando-se variáveis dummies específicas por países e tempo para representar os termos mencionados.

\footnotetext{
${ }^{11}$ Neste caso, o Teste de Hausman foi calculado a fim de justificar e dar maior respaldo à utilização das estimações com efeitos fixos. Os resultados deste teste encontram-se nas Tabelas 2 e 3 da seção de resultados.
} 
De acordo com Greene (2008), a presença de fatores não observados, neste caso os termos de resistência multilateral, correlacionados com as demais variáveis explicativas, aponta a estimação por efeitos fixos como a mais adequada. Pois, a estimação do modelo de efeitos fixos permite a inclusão dos termos de resistência multilateral como fatores não observados na equação empírica evitando o viés causado pela omissão dessas variáveis, que, de outro modo, estariam expressas no termo de erro da equação estimada. Portanto, o problema que ocorre quando esses termos são omitidos está relacionado à sua correlação com as proxies adicionadas no modelo como forma de representar os custos de transação não observados. Além disso, Shepherd (2008), em seu artigo intitulado "Notas Sobre a Teoria do Modelo Gravitacional para Comércio Internacional", reforça a importância de se estimar a equação de gravidade com efeitos fixos como forma de inclusão dos termos de resistência multilateral, o que não ocorreria em uma estimação com efeitos aleatórios, por exemplo.

Outros aspectos relevantes na estimação do modelo gravitacional referemse à presença de fluxos comerciais discrepantes, em razão da grande heterogeneidade dos padrões de comércio entre países e da presença de fluxos comerciais que são nulos (quando não há comercialização de determinado produto ou ausência de dados sobre tal fluxo). Os problemas resultantes dessas características dos dados são a provável ocorrência de heterocedasticidade e do viés de seleção amostral, que podem comprometer a qualidade das estimativas.

Em razão dessas características dos dados, há na literatura uma constante discussão sobre os melhores métodos de estimação do modelo gravitacional. Santos Silva \& Tenreyro (2006) destacaram que uma forma conveniente de contornar o problema da heterocedasticidade, além de incluir os fluxos zero na amostra, seria a estimação pelo método não linear Poisson Pseudo-MaximumLikelihood (PPML).

Desse modo, conforme sugestões de Anderson \& Van Wincoop (2004) e Santos Silva \& Tenreyro (2006), para avaliar a relação entre as diferentes variáveis do modelo proposto para o fluxo de comércio setorial brasileiro frente aos seus principais parceiros comerciais, utiliza-se, primeiramente, um modelo de efeitos fixos por MQO e, posteriormente, a estimação de um modelo de efeitos fixos utilizando-se o método PPML de Santos Silva e Tenreyro. A análise entre eles dá-se pela coerência dos sinais e pelos indicadores estatísticos de significância dos coeficientes, permitindo a comparação dos resultados entre os modelos, além de gerar um maior respaldo para a análise proposta.

\subsection{Fonte de dados}

Neste estudo foi utilizado um painel de dados referentes ao fluxo comercial entre o Brasil e seus principais parceiros comerciais (China, Estados Unidos, Argentina, Alemanha, Japão, Holanda e Chile), no intervalo anual de 1989 a 2011, para os setores agropecuário, químico, de metais e minerais, manufaturados e de máquinas e equipamentos de transporte. ${ }^{12}$ O painel de dados foi

\footnotetext{
${ }^{12}$ Os setores analisados neste trabalho seguem a metodologia da Classificação Uniforme do Comércio Internacional (CUCI) de dois dígitos, sendo que a composição de cada setor é a seguinte: agropecuário, composto por produtos alimentícios, animais vivos, bebidas, fumo, matéria prima não comestível, óleos, gorduras e ceras de origem animal e vegetal; metais e minerais, formado por minerais metálicos, mineral e fertilizante bruto, metais não ferrosos e sucata de metal; máquinas e equipamentos de transporte, constituído por máquinas e equipamentos industriais,
} 
obtido por meio do Trade Analysis and Information System (TRAINS) e do World Integrated Trade Solutions (WITS). Dessas fontes de dados foram extraídos os dados de comércio e tarifas.

Em relação aos dados do PIB e da taxa de câmbio real, estes foram obtidos por meio das International Financial Statistics (IFS) do International Monetary Fund (IMF). Já a variável distância utilizada referiu-se à distância em quilômetros entre as cidades mais populosas para cada par de países, essa variável foi obtida por meio dos dados do Centre D'Estudes Prospectives et d'Informations Internationales (CEPII). Para a tarifa, utilizou-se a tarifa média por setor, para cada país. Quanto à unidade de mensuração, os dados estão expressos dólares correntes.

\section{Resultados e discussão}

Nesta seção, a análise do comércio entre o Brasil e seus principais parceiros comerciais está divida em duas partes: a primeira corresponde à análise descritiva dos dados e a segunda consiste na avaliação e discussão dos resultados das estimativas do modelo empírico para os fluxos das exportações e importações setoriais.

\subsection{Análise descritiva dos dados}

A Tabela 1 apresenta as estatísticas descritivas gerais de cada uma das variáveis quantitativas que foram utilizadas no presente estudo. Os valores descritivos apresentados pelas distintas variáveis dependentes $\left(E x p_{i j}\right.$, t e $\left.I m p_{i j, t}\right)$ estão expressos em milhares de dólares norte-americanos e evidenciam o caráter heterogêneo do padrão de comércio setorial brasileiro. A média das exportações foi maior que a das importações, apresentando um valor superior a US\$ 1 bilhão e o valor máximo superior a US $\$ 26$ bilhões. Cabe ainda ressaltar que as variáveis relacionadas ao comércio, para todos os setores e parceiros selecionados, não apresentaram valor nulo e que o padrão da participação da renda dos países $i$ e $j$ na renda mundial, ou seja, a variável $\left(P I B_{i} P I B_{j, t}\right)$, também mostrou-se heterogênea entre os países.

Outra característica importante da amostra é o nível de proteção comercial representado pelas barreiras tarifárias (Tarif $a_{1 i j, t}$ e $\operatorname{Tarif}_{\operatorname{as}_{2 i j, t}}$ ). O uso das barreiras tarifárias parece ser mais praticado pelo Brasil, uma vez que os valores médio e máximo para a variável Tarif $a_{2 i j, t}, 14,84 \%$ e $51,76 \%$, foram maiores que a média e o valor máximo para a variável Tarif $a s_{1 i j, t}, 4,02 \%$ e $36,89 \%$. Quanto à variável distância $\left(\right.$ Dist $\left._{i j, t}\right)$, é importante destacar que foi utilizada a distância em quilômetros entre a cidade mais populosa do Brasil, São Paulo, e a cidade mais populosa de cada parceiro considerado na análise. A distância média entre o Brasil e seus parceiros comerciais ficou em torno de $9.685,51 \mathrm{~km}$.

Por fim, com relação às variáveis explicativas representativas da instabilidade da taxa de câmbio bilateral real de médio e longo $\operatorname{prazos}^{13}\left(U_{i j, t}\right)$ e do

equipamentos elétricos, ferroviários, aéreos e veículos rodoviários; químico, composto por produtos químicos orgânicos, inorgânicos, farmacêuticos, cosméticos e plásticos; e manufaturados, estabelecido por manufaturas utilizando metais, borracha, couro, madeira, papéis, fios têxteis e dispositivos elétricos.

${ }^{13}$ Nesta seção, a variável representante da Instabilidade da taxa de câmbio bilateral real de médio e longo prazo entre o Brasil e seus parceiros $\left(U_{i j, t}\right)$ será referida apenas como "instabilidade" 
Tabela 1: Médias, desvios-padrão e valores máximo e mínimo das variáveis que caracterizam a amostra utilizada na pesquisa, período 1989-2011

\begin{tabular}{lrrrr}
\hline Variável & \multicolumn{1}{c}{ Média } & Desvio Padrão & \multicolumn{1}{c}{ Mínimo } & \multicolumn{1}{c}{ Máximo } \\
\hline Exp $_{i j, t}$ & 1276685,0000 & 2204364,0000 & 6861,7600 & 26600000,0000 \\
Imp $p_{i j, t}$ & 1004610,0000 & 1845810,0000 & 291,7800 & 18000000,0000 \\
$P I B_{i} P I B_{j, t}$ & 2988980,0000 & 5422691,0000 & 12080,5300 & 37400000,0000 \\
Dist $_{i j, t}$ & 9685,5100 & 6094,6600 & 1691,0700 & 18549,6100 \\
Tarifas $_{i j, t}$ & 1,0402 & 0,0582 & 1,0000 & 1,3689 \\
Tarifas $_{i j, t}$ & 1,1484 & 0,0787 & 1,0143 & 1,5176 \\
$U_{i j, t}(2)$ & 0,1509 & 0,1430 & 0,0052 & 1,0707 \\
$U_{i j, t}(4)$ & 0,1748 & 0,1260 & 0,0253 & 0,7986 \\
$U_{i j, t}(6)$ & 0,1898 & 0,1125 & 0,0368 & 0,6569 \\
$U_{i j, t}(8)$ & 0,1976 & 0,1040 & 0,0436 & 0,6186 \\
$U_{i j, t}(10)$ & 0,2034 & 0,1006 & 0,0637 & 0,6047 \\
$U 3_{i j, t}(2)$ & 0,1402 & 0,0864 & 0,0201 & 0,4389 \\
$U 3_{i j, t}(4)$ & 0,1794 & 0,1018 & 0,0273 & 0,5500 \\
$U 3_{i j, t}(6)$ & 0,2027 & 0,0995 & 0,0249 & 0,5565 \\
$U 3_{i j, t}(8)$ & 0,2215 & 0,0969 & 0,0264 & 0,5335 \\
$U 3_{i j, t}(10)$ & 0,2351 & 0,0946 & 0,0275 & 0,5350 \\
\hline Fonte: Dado & & & &
\end{tabular}

Fonte: Dados da pesquisa.

Legenda: Expij,t são as exportações setoriais brasileiras para cada parceiro em análise; $I m p_{i j, t}$ são as importações setoriais brasileiras provenientes de cada parceiro; $P I B_{i} P I B_{j, t}$ é a multiplicação das rendas (PIB's) dos países i e j (ou seja, a participação da renda dos países i e j na renda mundial); $D i s t_{i j, t}$ é a distância entre os países i e j; Tarif $a s 1_{i j, t}$ - tarifa média efetivamente aplicada pelo importador sobre a exportação brasileira, dentro de cada setor; Tarif as $2_{i j, t}$ tarifa média efetivamente aplicada pelo Brasil sobre suas importações, dentro de cada setor; $U_{i j, t}$ é a variável representativa da instabilidade da taxa de câmbio bilateral real de médio e longo prazos (calculada com 2, 4, 6, 8 e 10 anos de defasagens); $U 3_{i j, t}$ é medida o efeito third country (mensurado com 2, 4, 6, 8 e 10 anos de defasagens).

efeito third country $\left(U_{3 i j, t}\right)$, pode-se observar, de modo geral, um padrão de comportamento entre as medidas com diferentes defasagens, indicando não haver problemas com a escolha do número de defasagem dessas variáveis. A análise comportamental da instabilidade cambial e do efeito third country será mais bem detalhada no próximo tópico dessa seção.

\subsection{Impactos da instabilidade cambial e do efeito third country sobre as exportações e importações setoriais brasileiras para seus principais parceiros}

Os resultados apresentados nas Tabelas 2 e 3 baseiam-se na estimação dos coeficientes da equação gravitacional empírica 4 por meio do modelo de Efeitos Fixos (dummies para países e tempo) por Mínimos Quadrados Ordinários (MQO) e pela estimação utilizando o método Poisson Pseudo-MaximumLikelihood (PPML) com Efeitos Fixos (EF). Esses procedimentos, utilizando-se dos métodos de estimação MQO e PPML, permitem a comparação dos resultados entre os modelos, além de gerar um maior respaldo para a análise proposta.

As estimativas dos coeficientes das variáveis dummies para países e tempo não foram apresentadas nas Tabelas 2 e 3, uma vez que não possuem interpre-

ou "incerteza" cambial. 
tação clara e seus resultados não são relevantes para as conclusões, servindo somente para evitar que seus efeitos afetem os coeficientes das demais variáveis. A adequação e a robustez dos resultados foram aferidas por diferentes estatísticas. A significância dos coeficientes do modelo de efeitos fixos por MQO foi examinada por meio do Teste F de Chow e o Teste de Wald verificou a significância conjunta das variáveis nas estimações quando se utilizou o método PPML. Adicionalmente, é importante destacar que a escolha do modelo de efeitos fixos, em relação ao de efeitos aleatórios, pode ser confirmada por meio do Teste de Hausman.

$\mathrm{Na}$ Tabela 2, os valores das exportações setoriais brasileiras para os sete parceiros comerciais considerados no estudo constituem a variável dependente, no período entre 1989 e 2011. De maneira geral, os resultados por MQO e PPML foram bons, com a maioria dos coeficientes estimados apresentado significância estatística e mostrando-se bastante similares em termos de sinais esperados para todas as variáveis explicativas. Além disso, por meio do Teste F e Teste Wald, a hipótese de que todos os coeficientes não explicam as exportações setoriais brasileiras foi rejeitada a $1 \%$ de significância nas estimações para todos os setores. Em relação ao Teste de Hausman, o mesmo também demonstrou-se estatisticamente significativo nas estimações para todos os setores analisados. Assim sendo, pode-se dizer que não foi possível rejeitar a hipótese nula de que efeitos de particularidades não observáveis dos países afetem as exportações setoriais do Brasil.

De acordo com os resultados para o setor agropecuário (Tabela 2), todas as variáveis apresentaram os sinais esperados para os dois métodos de estimação utilizados, sendo que a variável "Tarifas" não apresentou significância estatística para a estimação por MQO, mas, por outro lado, a estimação pelo método PPML comprovou a sensibilidade do setor ao impacto negativo das tarifas impostas às exportações agropecuárias brasileiras. Deve-se destacar, também, que a variável distância obteve significância estatística a 1\%, mas sua baixa magnitude revelou um fraco efeito sobre as exportações desse setor. Uma explicação plausível pode estar relacionada à forte vantagem comparativa que o país apresenta nos produtos agropecuários, o que compensaria os elevados custos de transporte para países mais distantes.

Com relação às variáveis instabilidade cambial e efeito third country, observou-se que ambas apresentaram uma relação inversa e estatisticamente significativa com os fluxos das exportações agropecuárias brasileiras. Destaca-se que a magnitude do efeito third country para esse setor foi a maior dentre os setores analisados. Esse resultado indica que uma maior instabilidade cambial de um terceiro país pode levar a uma maior redução das exportações agrícolas do Brasil para qualquer um dos parceiros dentre os sete países considerados.

Analisando os resultados para as exportações do setor de máquinas e equipamentos de transporte, verifica-se que esse setor foi o mais impactado pela variável distância, indicando que quanto maiores forem as distâncias entre o Brasil e seus parceiros, menores serão as quantidades de máquinas e equipamentos de transporte exportadas, pois maiores serão os custos de transporte. Ou seja, um aumento de $1 \%$ na distância (em $\mathrm{km}$ ), para o modelo MQO, geraria uma redução de $1,9 \%$ no volume exportado desse setor.

Para os setores de manufaturados, químico e o de metais e minerais, no que tange à variável utilizada para mensurar o tamanho do mercado nos países, neste caso, o produto dos PIB's, revelou um efeito positivo e significativo sobre as exportações dos três setores mencionados. Esse resultado indica, por 


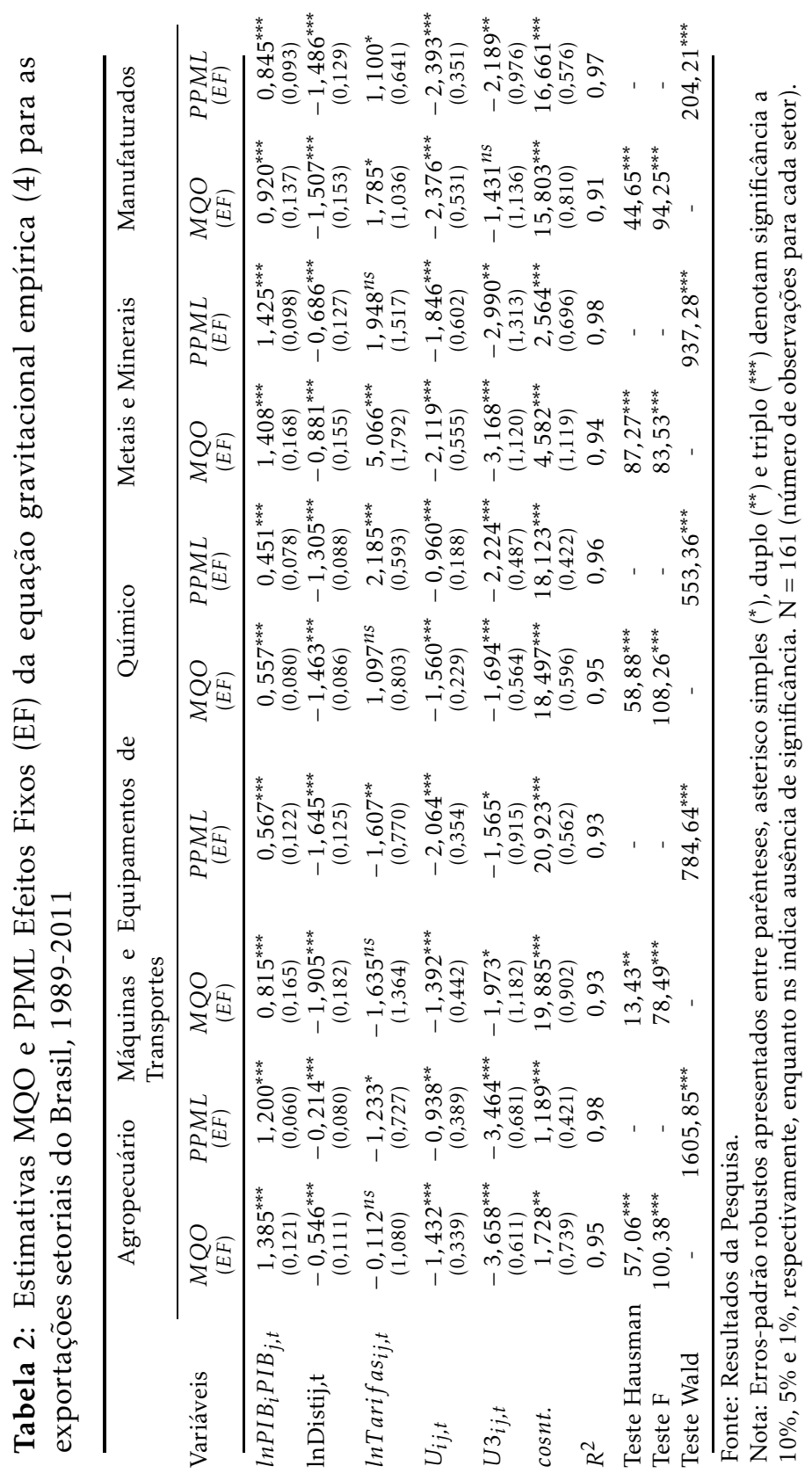


exemplo, que um aumento de $1 \%$ no nível de renda dos países, eleva em 1,4\% as exportações brasileiras de metais e minerais para os seus parceiros. Ainda em relação aos resultados dos três setores citados, é importante ressaltar que o sinal da variável usada para mensurar o efeito das barreiras tarifárias foi contrário ao esperado, além de não apresentar significância estatística em algumas estimações da Tabela 2. Esse efeito contrário das barreiras tarifárias também ocorreu no caso das estimativas para as importações brasileiras de produtos químicos (Tabela 3).

No entanto, vale destacar que outros trabalhos, utilizando estimações setoriais do modelo gravitacional, também encontraram sinais ambíguos e, muitas vezes, não significativos para as variáveis representativas das barreiras tarifárias. Corrêa (2011), por exemplo, ao analisar o impacto das barreiras tarifárias e não tarifárias sobre as exportações de produtos químicos do Brasil, encontrou sinal positivo e não significativo para a variável tarifa. A autora salienta que um dos fatores para tal resultado é a existência de muitos zeros pela inclusão de países pertencentes ao Mercosul.

No caso dos coeficientes estimados para a variável representativa da incerteza cambial $\left(U_{i j, t}\right)$ e do efeito third country $\left(U 3_{i j, t}\right)$, para os setores de produtos químicos, metais e minerais e o setor de produtos manufaturados, observa-se que as estimativas de tais variáveis revelaram-se estatisticamente significativas a $1 \%$, além de apresentar o sinal negativo esperado. Entretanto, ao revelar maiores valores negativos para o efeito third country, o setor de metais e minerais mostrou ser mais sensível ao impacto da instabilidade cambial de um terceiro país, pois uma elevação de $10 \%$ na volatilidade cambial entre os demais parceiros pode indicar uma redução de 6,9\% nas exportações brasileiras desse setor, segundo a estimativa do coeficiente $U 3_{i j, t}$ pelo método MQO com efeitos fixos. ${ }^{14}$ Já em relação à instabilidade cambial $\left(U_{i j, t}\right)$, o coeficiente estimado para tal variável apresentou o maior valor negativo para o setor de manufaturados, revelando que com um aumento de $10 \%$ na instabilidade cambial entre o Brasil e seus parceiros, as exportações brasileiras de manufaturados, para os sete países considerados, podem cair cerca de $4,5 \%$, segundo a estimativa do coeficiente $U_{i j, t}$ pelo método MQO com efeitos fixos. ${ }^{15}$

É importante mencionar que esses impactos negativos da instabilidade cambial e do efeito third country sobre o comércio setorial brasileiro também foram encontrados em outros estudos. Bittencourt (2004) analisou os efeitos da volatilidade da taxa de câmbio real e do efeito third country no comércio setorial entre o Brasil e os países do MERCOSUL. Os principais resultados encontrados pelo autor foram os impactos negativos da incerteza cambial sobre o comércio dos setores de produtos químicos, manufaturados e mineração, além de identificar um elevado impacto do efeito third country sobre o comércio do setor agrícola. Além desses resultados, o autor conclui que a falta de coordenação das políticas macroeconômicas adotadas, principalmente por Argentina e Brasil, tem sido uma ameaça ao futuro desse bloco econômico.

\footnotetext{
${ }^{14}$ Para obter a interpretação de elasticidade do efeito da 'instabilidade cambial' e do 'efeito third country' no comércio, por meio do modelo utilizado, deve-se multiplicar o coeficiente estimado pelo valor médio da medida utilizada na estimação. Desse modo, conforme as estatísticas presentes na Tabela 1, a média do efeito third country com 8 defasagens $\left[U 3_{i j, t}(8)\right]$ usada para obter esta interpretação foi 0,22 .

${ }^{15}$ Conforme as estatísticas descritivas presentes na Tabela 1, a média da variável instabilidade cambial com 8 defasagens $\left[U_{i j, t}(8)\right]$ usada para obter esta interpretação foi 0,19 .
} 
Pois, essas políticas desencontradas podem ser consideradas uma das causas da maior variabilidade cambial e de preços, o que traz impactos adversos ao comércio bilateral devido ao comportamento de aversão ao risco dos agentes econômicos.

Os resultados setoriais encontrados na Tabela 2 do presente estudo também são consistentes com os observados por Jesus (2010), que analisou o impacto da volatilidade cambial sobre as exportações setoriais brasileiras. A autora verificou, por meio de estimativas utilizando o modelo gravitacional, que as exportações do setor agropecuário foram as menos impactadas pela incerteza cambial, mas que, por outro lado, as exportações de tal setor também foram mais afetadas negativamente pela volatilidade cambial de um terceiro país. Outro resultado relevante encontrado pela autora foi a elevada influência negativa do efeito third country sobre as exportações do setor de manufaturados. Segundo a autora, esse resultado observado para o setor de manufaturados é justificado pelo alto grau de substituição do setor, visto que os produtos são muito similares. Assim, alterações na taxa de câmbio dos parceiros podem ocasionar grandes reduções nas exportações brasileiras.

A Tabela 3 apresenta as estimativas MQO e PPML Efeitos Fixos (EF) da equação gravitacional empírica 4 utilizando, como variável dependente, os valores das importações setoriais brasileiras provenientes dos sete parceiros comerciais considerados no estudo, no período entre 1989 e 2011. De modo geral, os resultados por MQO e PPML foram bons, com a maioria dos coeficientes estimados apresentando significância estatística e mostrando-se bastante similares em termos de sinais esperados para todas as variáveis explicativas estimadas pelos dois métodos. Além disso, por meio do Teste F e Teste Wald, a hipótese de que todos os coeficientes não explicam as importações setoriais brasileiras foi rejeitada a $1 \%$ de significância nas estimações para todos os setores, ou seja, pode-se afirmar que as variáveis são conjuntamente significantes. A respeito do Teste de Hausman, o mesmo revelou-se estatisticamente significativo nas estimações para todos os setores considerados. Dessa forma, pode-se dizer que não é possível rejeitar a hipótese nula de que efeitos de aspectos não observáveis dos países afetem as importações por setor do Brasil.

De acordo com os resultados para o setor agropecuário (Tabela 3), é importante ressaltar que a variável distância obteve significância estatística a $1 \%$ e o sinal negativo esperado, porém sua elevada magnitude demonstra maior impacto dos custos de transporte sobre as importações desse setor, ou seja, fato contrário ao ocorrido e comentado anteriormente em relação às exportações agropecuárias brasileiras (Tabela 2). Deve-se destacar, também, que a magnitude do efeito third country para o setor agropecuário foi a menor dentre as importações analisadas, resultado também contrário ao obtido na análise para as exportações. Uma provável explicação para a maior magnitude do efeito third country para as exportações do setor agropecuário é que, nos últimos anos, este setor tem se caracterizado pelo elevado ingresso de investimentos externos, desse modo, as variações cambiais dos demais parceiros têm afetado negativamente esses investimentos no setor, uma vez que o capital estrangeiro pode ficar mais caro ou melhor para ser aplicado em outro país, o que pode gerar uma redução na produção e um maior impacto negativo sobre as exportações do setor.

No caso dos coeficientes estimados para as importações do setor de máquinas e equipamentos de transporte, verifica-se que os resultados do efeito third country e da instabilidade cambial apresentaram significância estatística a $1 \%$ 


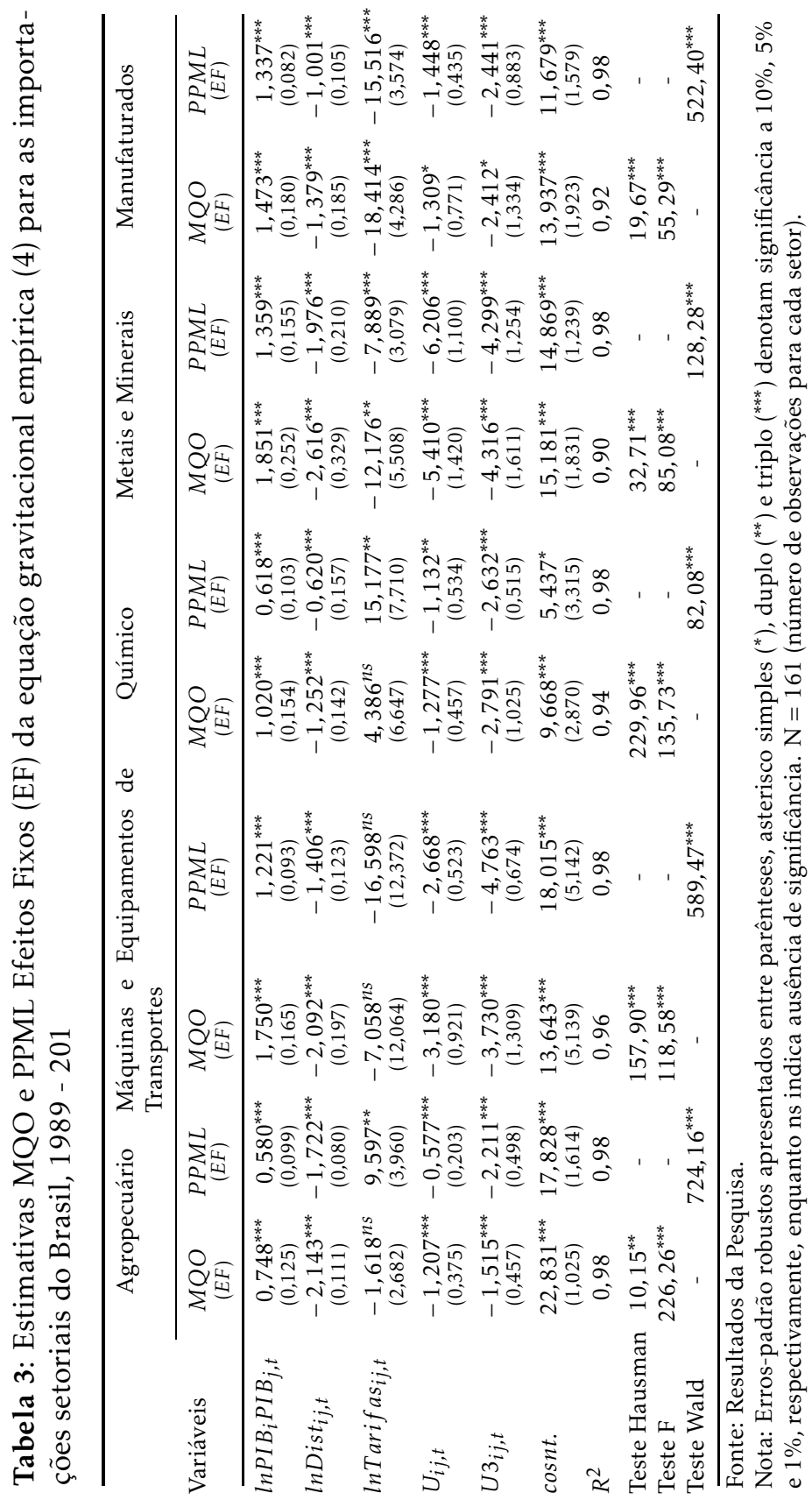


e foram negativamente relacionados com as importações do setor. Além disso, deve-se salientar que a magnitude das estimativas dessas variáveis foi elevada, só ficando abaixo dos resultados para o setor de metais e minerais. Com isso, evidencia-se que a incerteza cambial representa um importante obstáculo às importações deste setor, sugerindo que a falta de políticas macroeconômicas estáveis pode reduzir o comércio de máquinas e equipamentos de transporte.

Analisando as repostas para as variáveis explicativas em relação às importações do setor de produtos manufaturados, constata-se que as estimativas dos coeficientes para a variável tarifas, tanto pelo método dos MQO quanto pelo PPML, apresentaram o sinal negativo esperado e significância estatística a $1 \%$ e, também, revelaram ser os maiores valores, comparativamente aos resultados estimados dessa variável para os demais setores. Desse modo, as importações brasileiras de produtos manufaturados parecem ser muito sensíveis a mudanças tarifárias, pois uma redução de $1 \%$ nas tarifas médias do setor, para o modelo MQO, aumentaria as importações em $18 \%$, aproximadamente. Ainda em relação às importações do setor de manufaturados, pode-se observar que as demais variáveis também foram significativas estatisticamente e evidenciaram os sinais esperados.

Na tabela 3, quanto ao setor de metais e minerais, observa-se que todos os coeficientes estimados apresentaram o sinal esperado e foram estatisticamente significativos. Além disso, verifica-se que todos os resultados para o respectivo setor, com exceção dos coeficientes da variável tarifas, foram maiores em comparação aos dos demais setores analisados, indicando que todas as variáveis consideradas no modelo caracterizam-se como importantes determinantes do volume de importação brasileira de metais e minerais.

Portanto, é importante mencionar que o setor de metais e minerais demonstrou ser o mais impactado pelo conjunto de todas as variáveis explicativas, onde a variável representativa da incerteza cambial $\left(U_{i j, t}\right)$ e do efeito third country $\left(U 3_{i j, t}\right)$ foram as que revelaram os coeficientes de maior magnitude, principalmente, para a estimação pelo método dos MQO com efeitos fixos. Nesse sentido, uma elevação de $10 \%$ na volatilidade cambial entre os demais parceiros (efeito third country) pode indicar uma redução de $9,4 \%$ nas importações brasileiras desse setor, segundo a estimativa do coeficiente $U 3_{i j, t}$ pelo método MQO com efeitos fixos. ${ }^{16}$ Já em relação à instabilidade cambial, um aumento de $10 \%$ na volatilidade da taxa de câmbio bilateral real entre o Brasil e seus parceiros pode gerar uma redução de 10,2\% nas importações provenientes dos sete países considerados, segundo a estimativa do coeficiente $U_{i j, t}$ pelo método MQO com efeitos fixos. ${ }^{17}$

Esses resultados demonstram que as importações do setor de metais e minerais são bastante afetadas negativamente não só pela incerteza cambial da moeda brasileira, mas também pela volatilidade da taxa de câmbio entre os parceiros comerciais considerados. Este efeito pode estar ligado ao fato dos importadores realizarem mais contratos em moeda estrangeira e, assim, estarem mais vulneráveis às incertezas cambiais dos demais países. Desse modo, evidencia-se a ideia de que a busca por uma maior estabilidade nas políticas macroeconômicas brasileiras e dos parceiros analisados pode levar a uma re-

\footnotetext{
${ }^{16}$ Conforme as estatísticas descritivas presentes na Tabela 1, a média do efeito third country com 8 defasagens $\left[U 3_{i j, t}(8)\right]$ usada para obter esta interpretação foi 0,22 .

${ }^{17}$ Conforme as estatísticas descritivas presentes na Tabela 1 , a média da variável instabilidade cambial com 8 defasagens $\left[U_{i j, t}(8)\right]$ usada para obter esta interpretação foi 0,19
} 
dução dos efeitos adversos da instabilidade cambial no comércio desse setor e no dos demais.

Este impacto negativo da instabilidade cambial sobre os fluxos de importações setoriais também pode ser verificado no trabalho de Kafle (2011). No referido estudo, a autora buscou avaliar o efeito da volatilidade cambial e das taxas de câmbio bilaterais reais sobre as importações agrícolas e não agrícolas entre os Estados Unidos e os países da OCDE. Como esperado pela autora, o impacto da instabilidade cambial obteve efeito significativo e negativo em todos os tipos de importações analisados. No entanto, a magnitude do impacto foi maior sobre as importações agrícolas do que para as importações não agrícolas. Outros exemplos de trabalhos anteriores que estimaram o efeito sobre os fluxos de importações são os de Hooper \& Kohlhagen (1978) e Baek \& Koo (2009). Todos eles encontraram efeitos mistos da instabilidade cambial sobre os fluxos de importações.

É importante mencionar que a grande maioria dos estudos anteriores procurou estimar o efeito da inceteza cambial sobre os fluxos de comércio bilaterais totais ou sobre os fluxos de exportações entre os países. Desse modo, uma das principais contribuições do presente estudo é justamente a análise das estimativas da equação gravitacional empírica para as importações setoriais brasileiras, que, inclusive, obteve coeficientes estimados (Tabela 3) com variações e valores maiores do que as estimativas para as exportações setoriais do Brasil (Tabela 2), o que evidencia a relevância de estudos com análises desagregadas por setores e tipos de fluxo comercial.

Adicionalmente, as estimativas encontradas parecem estar de acordo com os resultados do trabalho de Aghion et al. (2009), o qual afirma que o efeito da instabilidade cambial sobre as firmas dos países dependerá do quão desenvolvido financeiramente é tal país e, também, quão dependente de investimentos iniciais e de liquidez essas firmas são. Portanto, de acordo com os resultados deste estudo, o impacto negativo da instabilidade cambial pode ser observado principalmente nos setores compostos por firmas que necessitam de maior liquidez e investimentos iniciais, como foi o caso do setor de máquinas e equipamentos de transportes, manufaturados e o de metais e minerais.

\section{Considerações finais}

Diante da evolução da participação das exportações e importações brasileiras no comércio internacional e do cenário de incertezas cambiais, o presente estudo se propôs a avaliar como a instabilidade cambial de médio/longo prazo tem afetado os fluxos de exportações e importações setoriais do Brasil com seus principais parceiros comerciais, no período de 1989 a 2011. Os resultados obtidos por essa análise evidenciam que tanto as exportações quanto as importações setoriais entre o Brasil e seus principais parceiros são negativamente afetadas não só pela própria incerteza cambial, mas também pela instabilidade da taxa de câmbio dos parceiros em análise. Desse modo, podese dizer que foi aceita a hipótese de que a instabilidade da taxa de câmbio bilateral real de médio/longo prazo tem influenciado negativamente os fluxos comerciais setoriais do Brasil com seus parceiros considerados.

Portanto, com base nos resultados de estudos correlatos, verifica-se que os impactos diferenciados por setor e por fluxo de comércio, quando considerada a instabilidade cambial do país e dos parceiros, podem ser decorrentes de 
um encarecimento do capital estrangeiro demandado por setores que necessitam de elevados níveis de investimentos iniciais, como o setor de máquinas e equipamentos, metais e minerais e manufaturados, consequência de um setor financeiro em desenvolvimento, ou no caso dos fluxos comerciais como um todo, consequência da incerteza que faz com que os empresários passem a investir em setores para os quais possuem maior conhecimento de mercado e menores riscos, ou simplesmente a preferência em priorizar o mercado doméstico.

Diante disso, a estabilidade da economia e a tomada de atitudes que reduzam as incertezas sobre os movimentos da taxa de câmbio são importantes variáveis a serem consideradas, caso o Brasil e seus principais parceiros analisados almejem elevar os fluxos comerciais bilaterais.

Um argumento nesse sentido, nos anos posteriores à crise internacional de 2008, foram os debates econômicos concentrados nas tensões acerca da desvalorização das moedas nacionais, cenário batizado como "batalha cambial". Protagonizado por EUA e China, o fenômeno repercutiu fortemente nos demais países. Pois, a desvalorização da moeda chinesa vinha constituindo objeto de controvérsias entre os países, especialmente devido a seus efeitos sobre o comércio mundial.

Outro ponto importante é busca por uma maior coordenação das políticas macroeconômicas adotadas pelos países membros do MERCOSUL, principalmente Brasil e Argentina. Pois, políticas desencontradas podem ser consideradas uma das causas da maior variabilidade cambial e de preços dentro dos blocos econômicos.

Para futuros trabalhos, sugerem-se a utilização de outros métodos de estimação, uso de outras medidas da incerteza cambial, além da incorporação de um número maior de países.

\section{Referências Bibliográficas}

Africano, P. A. \& Magalhães, M. (2005), 'Fdi and trade in portugal: A gravity analysis', FEP Working Papers (174), 1-25.

Aghion, P., Bacchetta, P., Ranciére, R. \& Rogoff, K. (2009), 'Exchange rate volatility and productivity growth: The role of financial development.', Journal of Monetary Economics 56(4), 494-513.

Anderson, J. E. \& Van Wincoop, E. (2003), 'Gravity with gravitas: a solution to the border puzzle', American Economic Review 93(1), 170-192.

Anderson, J. E. \& Van Wincoop, E. (2004), 'Trade costs', Journal of Economic Literature 42(3), 691-751.

Associação de Comércio Exterior do Brasil (2012), 'Radiografia do comércio exterior brasileiro: passado, presente e futuro'.

URL: Disponível em: www.aeb.org.br/userfiles/file/AEB - Radiografia Comércio Exterior Brasil.pdf. Acesso em: mar/2012.

Baek, J. \& Koo, W. (2009), 'Assessing the exchange rate sensitivity of u.s. bilateral agricultural trade', Canadian Journal of Agricultural Economics 57(2), 187-203. 
Baier, S. L. \& Bergstrand, J. H. (2001), 'The growth of world trade: Tariffs, transport costs, and income similarity', Journal of International Economics 53(1), 1-27.

Bittencourt, M. V. L. (2004), The Impacts of Trade Liberalization and Macroeconomic Instability on the Brazilian Economy, Ph.d. dissertation, The Ohio State University.

Chit, M. M., Rizov, M. \& Willenbrocked, D. (2010), 'Exchange rate volatility and exports: New empirical evidence from the emerging east asian economies', The World Economy 33(2), 239-263.

Cho, G., Sheldon, I. M. \& Mccorriston, S. (2002), 'Exchange rate uncertainty and agricultural trade', American Journal of Agricultural Economics 84(4), 932-942.

Corrêa, C. R. (2011), Impactos das notificações ao acordo de barreiras técnicas nas exportações de produtos químicos do brasil, Master dissertation, Universidade Federal de Viçosa, Viçosa, MG.

Côté, A. (1994), 'Exchange rate volatility and trade', Working Paper of Bank of Canada 94(5), 1-28. http://www.bankofcanada.ca/wpcontent/uploads/2010/04/wp94-5.pdf.

De Grauwe, P. \& De Bellefroid, B. (1987), Long-run exchange rate variability and international trade, in S. Arndt \& J. Richardson, eds, 'Real-financial Linkages Among Open Economies’, MIT Press, Cambridge, MA, pp. 193-212.

De Grauwe, P. \& Skudelny, F. (2000), 'The impact of emu on trade flows', Weltwirtschaftliches Archiv 136(3), 388-402.

Dell'ariccia, G. (1999), 'Exchange rate fluctuations and trade flows: Evidence from the european union', IMF Staff Papers 46(3), 315-334.

Dellas, H. \& B., Z. (1993), 'Real exchange rate volatility and international trade: a reexamination of the theory', Southern Economic Journal 59(2), 651657.

Farrell, V., Rosa, D. \& Mccown, T. A. (1983), 'Effects of exchange rate variability on international trade and other economic variables: a review of the literature', Board of Governors of the Federal Reserve System. Staff Studies n. 130.

Greene, W. H. (2008), Econometric Analysis, 6th edn, Pearson Education Inc.

Hooper, P. \& Kohlhagen, S. (1978), 'The effect of exchange rate uncertainty on the prices and volume of international trade', Journal of International Economics 8(4), 483-511.

Jesus, L. (2010), O impacto da volatilidade cambial nas exportações brasileiras para o mercosul: uma análise em painel dinâmico, Master dissertation, Universidade Federal do Paraná, Curitiba, PR.

Jozsef, F. (2011), 'The effect of exchange rate volatility upon foreign trade of hungarian agricultural products', Studies in Agricultural Economics 113(1), 85-96. 
Kafle, K. R. (2011), Exchange rate volatility and bilateral agricultural trade flows: The case of the united states and oecd countries, Master dissertation, Louisiana State University, The Department of Agricultural Economics and Agribusiness.

Kenen, P. \& Rodrik, D. (1986), 'Measuring and analysing the effects of shortterm volatility in real exchange rates', Review of Economics and Statistics 68(1), 311-315.

Krugman, P. (1980), 'Scale economies, product differentiation, and the pattern of trade', American Economic Review 70(5), 950-959.

Kume, H. \& Piani, G. (2000), 'Fluxos bilaterais de comércio e blocos regionais: uma aplicação do modelo gravitacional', IPEA. Texto para Discussão, n.749, Rio de Janeiro: IPEA.

Mendonça, T. G. (2011), 'Instituições e comércio bilateral de produtos agropecuários', $39^{\circ}$ Encontro Nacional de Economia.

URL: Disponivel em: <http://anpec.org.br/encontro/2011/- inscricao/arquivos/0005e19ded7bc83bcfb76b1a75f118b75ab.doc>. Acesso em: Mar/2012.

Peree, E. \& Steinherr, A. (1989), 'Exchange rate uncertainty and foreign trade', European Economic Review 33(6), 1241-1264.

Rose, A. (2000), 'One money, one market: the effect of common currencies on trade', Economic Policy 15(30), 7-46.

Santos Silva, J. M. C. \& Tenreyro, S. (2006), 'The log of gravity', The Review of Economics and Statistics 88(4), 641-658.

Shepherd, B. (2008), 'Notes on the "theoretical" gravity model of international trade', Niehaus Center, Princeton University \& GEM, Sciences Po.

Souza, F. E. P. \& Hoff, C. R. (2003), 'O regime cambial brasileiro: flutuação genuína ou medo de flutuação?', $31^{\circ}$ Encontro Nacional de Economia. Anais... Porto Seguro, BA: ANPEC, 2003. CD-ROM.

Uncomtrade (2012), 'Pesquisa econômica - dados estatísticos', United Nations Commodity Trade Statistics Database. Disponível em: www.comtrade.un.org. Acesso em: Set./2012.

Vasconcellos, M. A., Gremaud, A. P. \& Toneto, R. (1996), Economia brasileira contemporânea, 3 edn, Atlas. 